\title{
A Multinational Longitudinal Study Incorporating Intensive Methods to Examine Caregiver Experiences in the Context of Chronic Health Conditions: Protocol of the ENTWINE-iCohort
}

\author{
Val Morrison ${ }^{1, *(D)}$, Mikołaj Zarzycki ${ }^{1}$, Noa Vilchinsky ${ }^{2}$, Robbert Sanderman ${ }^{3}$, Giovanni Lamura ${ }^{4}$, \\ Oliver Fisher ${ }^{4,5}{ }^{(D}$, Giulia Ferraris ${ }^{3}$ (D), Saif Elayan ${ }^{6}$, Erik Buskens ${ }^{7}$, Eva Bei ${ }^{2}$, Anne Looijmans ${ }^{3}$, \\ Viola Angelini ${ }^{6}(\mathbb{D})$ and Mariët Hagedoorn ${ }^{3}$
}

check for updates

Citation: Morrison, V.; Zarzycki, M.; Vilchinsky, N.; Sanderman, R.; Lamura, G.; Fisher, O.; Ferraris, G.; Elayan, S.; Buskens, E.; Bei, E.; et al. A Multinational Longitudinal Study Incorporating Intensive Methods to Examine Caregiver Experiences in the Context of Chronic Health Conditions: Protocol of the ENTWINE-iCohort. Int. J. Environ. Res. Public Health 2022, 19, 821. https://doi.org/10.3390/ ijerph19020821

Academic Editor: Ko-Ling Chan

Received: 29 November 2021

Accepted: 8 January 2022

Published: 12 January 2022

Publisher's Note: MDPI stays neutral with regard to jurisdictional claims in published maps and institutional affiliations.

Copyright: (c) 2022 by the authors Licensee MDPI, Basel, Switzerland. This article is an open access article distributed under the terms and conditions of the Creative Commons Attribution (CC BY) license (https:// creativecommons.org/licenses/by/ $4.0 /)$.
1 School of Human and Behavioural Sciences, Bangor University, Bangor LL57 2AS, UK; zarzycki@bangor.ac.uk

2 Department of Psychology, Faculty of Social Sciences, Bar-Ilan Univeristy, Ramat Gan 5290002, Israel; noa.vilchinsky@biu.ac.il (N.V.); eva.bei@biu.ac.il (E.B.)

3 Department of Health Psychology, University Medical Center Groningen, University of Groningen, 9713 GZ Groningen, The Netherlands; r.sanderman@umcg.nl (R.S.); g.m.a.ferraris@umcg.nl (G.F.); a.looijmans@umcg.nl (A.L.); mariet.hagedoorn@umcg.nl (M.H.)

4 Centre for Socio-Economic Research on Ageing, IRCCS INRCA-National Institute of Health and Science on Ageing, 60124 Ancona, Italy; g.lamura@incra.it (G.L.); o.fisher@inrca.it (O.F.)

5 Department of Economics and Social Sciences, Università Politecnica delle Marche, 60121 Ancona, Italy

6 Department of Economics, Econometrics and Finance, Faculty of Economics and Business, University of Groningen, Nettelbosje 2, 9747 AE Groningen, The Netherlands; s.y.i.elayan@rug.nl (S.E.); v.angelini@rug.nl (V.A.)

7 Faculty of Medical Sciences, Antonius Deusinglaan 1, 9713 AV Groningen, The Netherlands; e.buskens@umcg.nl

* Correspondence: v.morrison@bangor.ac.uk; Tel.: +44-1248-382485

\begin{abstract}
Informal caregivers are those who provide unpaid care to a relative or friend with a chronic illness, disability or other long-lasting health or care need. Providing informal care in the context of chronic health conditions presents a significant global challenge. Examination of the determinants of informal caregivers' behaviour, especially in terms of motivations and willingness to provide/receive care, is crucial to understanding the nature of caregiver and care recipient experiences. A large group of international researchers have co-operated to execute the ENTWINE iCohort-a multinational, transdisciplinary, longitudinal study incorporating intensive methods to examine caregiver experiences in the context of chronic health conditions. The aim of ENTWINE-iCohort is to investigate the broad spectrum of factors, i.e., cultural, personal, geographical, relational, psychological, and economic that may affect motivations, willingness to provide or receive care, among diverse groups of informal caregivers and their care recipients, in different countries that have different care systems. Study questionnaires will be disseminated on-line in nine countries: Germany, Greece, Ireland, Italy, Israel, the Netherlands, Poland, Sweden, and the UK. Cross-sectional and longitudinal multivariate analysis, including intensive longitudinal and dyadic data analysis will be applied to examine the relative contribution of the above factors to caregiver or care recipient wellbeing.
\end{abstract}

Keywords: informal caregiving; multinational; caregiver motivations; wellbeing

\section{Introduction}

Due to medical advancements and the subsequent rise in longevity worldwide, societies everywhere are facing a growing ageing population with an increase in those living with chronic, often disabling health conditions. However, in spite of this, many countries, such as the United Kingdom, The Netherlands and Italy, have seen a decline in spending on social care over the past decade [1,2]. Although investment has taken place more recently adult social care is considered to be in crisis and a significant funding gap exists [1,2]. Whilst investment in social care services fails to meet demand, families are required to 
manage care more informally, with the burden of care typically falling to female family members $[3,4]$. This continuing gender imbalance is in spite of an increased percentage of women who are also in paid employment [5,6]. This means that where the needs of older/ill individuals for long-term care is rising, the availability of informal caregivers is in decline. Known as the "Care Gap", this will create huge problems for the sustainability of global health care systems that rely heavily on the provision of informal care. Thus, a multinational analysis of informal caregivers' willingness to care and putative determinants of it as well as of informal caregivers' wellbeing and other outcomes is essential for informing care solutions and public policy.

A large group of international researchers have co-operated to execute the ENTWINE iCohort-a multinational, transdisciplinary longitudinal study incorporating intensive methods to examine caregiver experiences in the context of chronic health conditions. This paper presents the study protocol.

An informal caregiver for ENTWINE purposes is defined as a family member or friend who adopts a caring role for a person with a chronic health condition, disability, or other care need where needs require the caregiver to perform tasks beyond those typical of their usual role/relationship.

Research has commonly identified love and affection as the primary motivation for adopting a typically unpaid caregiver role [7,8] along with motives of duty and reciprocity [9]. However the complexity of dyadic relationships and attachments [10-14], the existence of varying cultural norms, values and obligations [15,16], competing social and economic demands on potential caregivers [17] and the fact that modern families tend not to be as geographically co-located as in previous generations [18-22] are less typically examined in relation to their influence on caregiver motivations. We face major challenges if we are to meet the demand for care (including informal care) across Europe on a societal (and often familial) assumption of family member willingness to adopt a caregiving role in the home or community. There is a real risk that the numbers experiencing negative physical and mental wellbeing as a consequence of caregiving [23-27] will increase and/or that the willingness to care will subside as people are expected to work for longer [28-30].

If informal care is to be maintained and supported, we need to better understand the range of factors-personal, interpersonal, social, economic, and geographic - that may influence caregiver willingness and motivation to adopt and maintain this demanding role or which may mitigate against negative care outcomes. The evidence gap that this study seeks to address will be informed by data from multiple countries facing similar ageing demographics but with different cultural and social norms around illness and care, and different care systems. Such understanding is important to the creation of culturally relevant policy and practice.

\subsection{Primary Objective}

To examine the current experience of caregiving, motivations to care and influences thereon among a diverse group of informal caregivers recruited by means of an intensive longitudinal cohort study (ENTWINE-iCohort) conducted in the five countries represented in the ENTWINE Consortium network (UK, Ireland, The Netherlands, Italy and Israel) plus four other EU countries (Germany, Greece, Poland, Sweden).

\subsection{Secondary Objectives}

To conduct a full examination of the:

Determinants of, and cultural differences in, motivations and willingness to provide care with specific attention paid to the contribution of personal values and meanings attached to illness and caregiving and their impact on caregiver outcomes,

Personal and geographical barriers and facilitators of caregiving with specific attention paid to the contribution of caregivers' personality traits and geographic distance from the care recipient upon willingness to care and caregiver outcomes, 
Diversity in experiences of caregiver-care recipient dyads, with specific attention paid to the characteristics of the dyads, interpersonal processes, willingness to provide and receive care and individual and relational outcomes.

Patterns of and differences across countries in informal care costs, as well as the preferences for, and use of, formal versus informal caregiving, and the personal and contextual influences thereon.

Characteristic of access to, use of and challenges of household-based migrant care work and how this supports informal caregiving

Each of these objectives is addressed in detail by one early stage researcher (five in total), each under supervision of two senior academics, and with oversight held by a Work Package Lead and ENTWINE management team.

\section{Materials and Methods}

Full study design:

The ENTWINE iCohort study shall combine:

(i) longitudinal data collection (Baseline +6 months follow-up) using an electronic survey tool with measures and items addressing the project objectives, with a sample of informal caregivers;

(ii) longitudinal data collection (Baseline +6 months) using an electronic survey tool containing a subset of measures and items addressing the project objectives, with a sample of care recipients;

(iii) a weekly assessment component to examine change over time using a subset of measures employed with caregivers and care recipients.

Setting:

Countries directly involved in the WorkPackage, ENTWINE iCohort included the UK (Morrison, Zarzycki); The Netherlands (Hagedoorn; Buskens, Ferraris, Elayan); Israel (Vilchinsky, Bei); and Italy (Lamura, Fisher) and the early stage researchers appointed also brought representation from Greece and Poland. Three further countries joined as collaborators based on targeting geographical diversity across Europe (Ireland, Germany, Sweden), and thus the setting was inclusive of northern, southern, eastern and western Europe, and affiliated country Israel.

The QUESTBACK online system was selected for collecting and storing data for the ENTWINE iCohort. Questback EFS (Enterprise Feedback Suite) is recognized by the Coordinating Centre UMCG as a secure data sharing tool. Data in EFS is only accessible by the members of the research team and consists of the e-mail address participants provide, their code and the data collected in the questionnaires. As such, this platform was considered suitable for our multinational study.

The Sample Selection Inclusion/Exclusion

There will be two samples: Caregivers and a care recipient sub-sample to enable dyadic analyses for those matched to a caregiver.

Inclusion criteria:

To be eligible, caregivers must:

Be aged over 18 years old;

Be providing care for a family member or friend with a chronic health condition, disability or any other care need;

Provide self-declared cognitive capacity to complete questionnaires (brief screener included to check for cognitive impairment);

Have access to the Internet.

The secondary sample is that of care recipients, identified either by a participating caregiver as receiving their care, or through direct recruitment advertising. To be eligible, care recipients must

Be aged over 18 years old;

Have a caregiver declaration regarding care recipient cognitive and physical capacity to complete questionnaires online; 
Have access to the Internet.

\section{Recruitment Methods}

Caregivers and care recipients are invited to participate jointly in the intensive longitudinal cohort study, but caregivers are encouraged to participate even where the care recipient is unable/unwilling to participate.

Recruitment will be either by (1) broad-based online recruitment via academic, social and news media and care or patient relevant organisations or (2) a more targeted, direct approach via user groups, carer organisations and primary and secondary care settings using advertising (poster displays, flier distribution) and direct face-to-face contact (due to COVID-19 restrictions direct face-to-face recruitment was not initiated). For recruitment method 1 a multi-language advertisement/flier was produced for all included study sites inviting adults who meet the aforementioned eligibility (inclusion) criteria to, for example,

"Take part in an international study of caregiving-find out more at this URL for ENTWINE iCohort survey link" (see Supplementary File S1).

These fliers shall be distributed in each country via national and international charity organisations specific to our target conditions, e.g., Alzheimer's Society, Stroke Association, Eurocarers (and national equivalents) and, subject to local approvals, also displayed in public venues such as community pharmacists, health, leisure, or educational centres.

The flier/advertisement will direct potential caregiver participants to the online platform for the ENTWINE-iCohort where they can register and access an eligibility survey (Supplementary File S11), a full Participant Information Sheet (PIS, Supplementary File S2) and electronic consent (Consent Form, Supplementary File S3) shall be required before the caregiver survey (baseline or follow-up, or the intensive longitudinal component, Supplementary Files S4, S6 and S8) can be accessed.

The caregiver (CG) shall be invited to consider whether they wish to nominate their care recipient (CR) to take part in the parallel Care Recipient Survey (baseline or follow-up, or the intensive longitudinal component, Supplementary Files S5, S7 and S9) and if so they shall direct them to the relevant registration site where they can access the CR survey tool for independent completion. CRs who self-identify will also be directed to the relevant registration site to access the CR survey tool. Both CG and CR will also be asked to indicate willingness to be contacted for a future related research study (Supplementary File S10).

\section{Questionnaire Randomisation}

Caregivers: the questionnaire for the CG is divided into four modules (Note that the economic, employment and migrant care work sub-components were combined into one module although this addresses the work of 2 ESRs), that is a core module and one module for variables that are specific for each sub-study $(1=$ cultural aspects; 2 = personality and geographical barriers; 3 = interpersonal processes; 4 = employment and costs including migrant care work). The core module includes personal and demographic information, care context and care task information, willingness to care and wellbeing outcomes (affect, gains, burden). In addition to the core module, participants will randomly receive two other modules (see Figure 1) as programmed within Questback.

This process will reduce the burden for participants and ensures that drop-out and missing values do not centre on specific modules (i.e., if a module is always offered last, this could increase the chance of missing data for this specific module).

Care recipients. The questionnaire for the CR is much shorter and the same for all CRs i.e., there are no modules. 


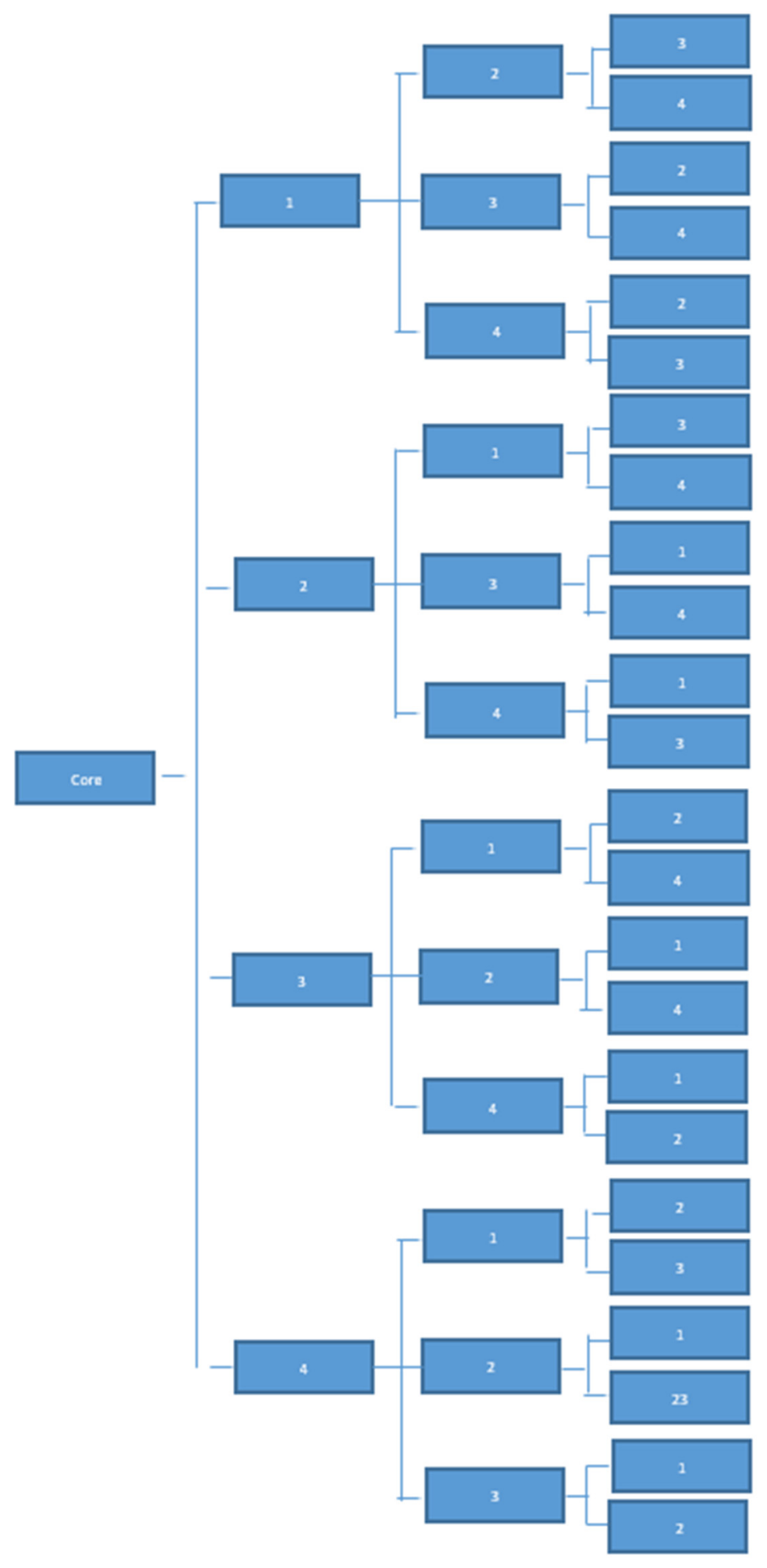

Figure 1. Questback randomization process. Note: $1=$ cultural and psychosocial aspects; 2 = personality and geographical barriers; 3 = interpersonal processes; 4 = employment, costs and migrant care work.

\section{Sample Size Calculation}

Whilst each specific ESR project has its own objectives (as described above), three are addressing those in relation to the primary outcomes of willingness and motivations to care. Given the number of variables to be assessed (see Measures, approximately 30 variables will be used in total) it is our intention to recruit CGs to the case: variable ratio of 10:1, 
i.e., 300 CGs per country. For CRs, who are assessed on fewer variables, we expect at least 150 participants per country-anticipating that at least $50 \%$ of our sample of 300 CGs per country will identify an eligible care recipient (based on our previous research). Analyses will be appropriate to the attained sample size on study completion in order to achieve satisfactory statistical power.

The a priori sample size calculation was informed by previous research. Achieving $90 \%$ power to detect a medium sized association/ explained variance $R^{2}=0.25$, with entry of up to 10 independent variables (randomly selected variables dependent on prior analyses) and willingness to care (measured as a continuous variable) in a multivariate regression analysis with an alpha of 5\% (G*Power 3.1, [31]).

\section{Measures}

Table 1 presents all measures used within the ENTWINE iCohort survey, with a brief description of the concept addressed, timings of use, and whether they reflect a key outcome measure (DV) or an IV/potential mediator or moderator variable. Wherever possible, validated instruments were employed, and short-form assessments were preferable where available in order to reduce participant burden.

Table 1. Summary of measures.

\begin{tabular}{|c|c|c|c|c|c|c|c|}
\hline Section/Measure & $\begin{array}{l}\text { Description and } \operatorname{Max}^{*} \mathbf{N} \\
\text { of Items }\end{array}$ & Scoring & $\begin{array}{c}\text { IV, DV, } \\
\text { Mod, Med }\end{array}$ & $\begin{array}{c}\mathrm{CG} \\
\text { Baseline }\end{array}$ & $\begin{array}{c}\text { CG } \\
\text { Follow Up }\end{array}$ & $\begin{array}{c}\text { CR } \\
\text { BASELINE }\end{array}$ & $\begin{array}{l}\text { CR Follow } \\
\text { Up }\end{array}$ \\
\hline \multicolumn{8}{|c|}{ Core } \\
\hline $\begin{array}{c}\text { Caregiver } \\
\text { Self-report } \\
\text { Personal and } \\
\text { Demographic } \\
\text { Questionnaire }\end{array}$ & $\begin{array}{l}\text { Seventy four (74) items } \\
\text { addressing age, gender, } \\
\text { home location, } \\
\text { partnership status, } \\
\text { relationship type, } \\
\text { religion, ethnicity, } \\
\text { household composition, } \\
\text { highest level of attained } \\
\text { education, employment } \\
\text { status, income, other } \\
\text { dependents, own health } \\
\text { condition, health } \\
\text { condition of care } \\
\text { recipient, length of illness, } \\
\text { distance from caregiver } \\
\text { to care recipient, } \\
\text { treatment or medication, }\end{array}$ & Categorical responses & $\begin{array}{l}\text { IV, Mod, } \\
\text { Med }\end{array}$ & $\checkmark$ & $\boldsymbol{\nu}$ & $x$ & $x$ \\
\hline $\begin{array}{l}\text { Care Recipient } \\
\text { Self-report } \\
\text { Personal and } \\
\text { Demographic } \\
\text { Questionnaire }\end{array}$ & $\begin{array}{l}\text { Fifty eight (58) items } \\
\text { addressing age, gender, } \\
\text { home location, } \\
\text { partnership status, } \\
\text { relationship type, } \\
\text { religion, ethnicity, highest } \\
\text { level of attained } \\
\text { education, employment } \\
\text { status, income, other } \\
\text { dependents, own health } \\
\text { condition, health } \\
\text { condition of care } \\
\text { recipient, length of } \\
\text { illness, distance from } \\
\text { caregiver to care recipient }\end{array}$ & Categorical responses & $\mathrm{IV}, \mathrm{Mo}, \mathrm{Me}$ & $x$ & $x$ & $\checkmark$ & $\checkmark$ \\
\hline
\end{tabular}


Table 1. Cont.

\begin{tabular}{|c|c|c|c|c|c|c|c|}
\hline Section/Measure & $\begin{array}{l}\text { Description and } \mathrm{Max}^{*} \mathrm{~N} \\
\text { of Items }\end{array}$ & Scoring & $\begin{array}{c}\text { IV, DV, } \\
\text { Mod, Med }\end{array}$ & $\begin{array}{c}\text { CG } \\
\text { Baseline }\end{array}$ & $\begin{array}{c}\text { CG } \\
\text { Follow Up }\end{array}$ & $\begin{array}{c}\text { CR } \\
\text { BASELINE }\end{array}$ & $\begin{array}{l}\text { CR Follow } \\
\text { Up }\end{array}$ \\
\hline $\begin{array}{l}\text { Inclusion of } \\
\text { Other Self Scale } \\
\text { (IOS) [32] }\end{array}$ & $\begin{array}{l}\text { One item assesses the } \\
\text { extent to which } \\
\text { participants include } \\
\text { another person in their } \\
\text { sense of self }\end{array}$ & $\begin{array}{l}\text { 7-point Likert scale in } \\
\text { which participants } \\
\text { choose a pair of circles } \\
\text { from seven with } \\
\text { different degrees of } \\
\text { overlap (ranging from } \\
1=\text { no overlap; to } \\
7=\text { most overlap) } \\
\text { Higher scores indicate } \\
\text { greater inclusion of } \\
\text { other in self }\end{array}$ & $\begin{array}{l}\text { IV, Mod, } \\
\text { Med }\end{array}$ & $\checkmark$ & $\checkmark$ & $\checkmark$ & $\checkmark$ \\
\hline Katz ADL [33] & $\begin{array}{l}\text { Six items assess } \\
\text { functional status via } \\
\text { measurement of the } \\
\text { person's ability to } \\
\text { perform activities of daily } \\
\text { living independently }\end{array}$ & $\begin{array}{c}\text { Items address } \\
\text { independence yes (1), } \\
\text { no (0) in performing } \\
\text { bathing, dressing, } \\
\text { toileting, transferring, } \\
\text { continence, and } \\
\text { feeding. } \\
\text { A score of } 6 \text { indicates } \\
\text { full function, } 4 \\
\text { indicates moderate } \\
\text { impairment, and } 2 \text { or } \\
\text { less indicates severe } \\
\text { functional impairment. }\end{array}$ & IV & $\checkmark$ & $\checkmark$ & $\checkmark$ & $\checkmark$ \\
\hline $\begin{array}{l}\text { COVID-19 } \\
\text { related questions }\end{array}$ & $\begin{array}{l}\text { Ten (10) items address the } \\
\text { impact of COVID-19 on } \\
\text { employment, access to } \\
\text { support services, } \\
\text { willingness to care, } \\
\text { provision of practical, } \\
\text { emotional, personal care }\end{array}$ & $\begin{array}{l}\text { Numerical and } \\
\text { categorical }\end{array}$ & Med, Mod & $\checkmark$ & $\checkmark$ & $x$ & $x$ \\
\hline $\begin{array}{l}\text { Use of paid } \\
\text { home care } \\
\text { services and their } \\
\text { characteristics }\end{array}$ & $\begin{array}{l}\text { Thirty five (35) items in } \\
\text { the caregiver baseline } \\
\text { and follow up and } 37 \\
\text { items in the care recipient } \\
\text { baseline and follow up } \\
\text { assess the self-reported } \\
\text { use of paid home care } \\
\text { services by the care } \\
\text { recipient. Demographics } \\
\text { of paid care workers i.e., } \\
\text { gender, age, nationality, } \\
\text { migration background, } \\
\text { live-in or live-out }\end{array}$ & $\begin{array}{l}\text { Numerical and } \\
\text { categorical }\end{array}$ & IV, DV & $\checkmark$ & $\checkmark$ & $\checkmark$ & $\checkmark$ \\
\hline Financial benefits & $\begin{array}{l}\text { Six items regarding: cash } \\
\text { benefits, financial } \\
\text { compensation during } \\
\text { care leave, tax benefits } \\
\text { e.g., exemptions, } \\
\text { deductions, credits, } \\
\text { coverage of social or } \\
\text { pension contributions, } \\
\text { caregiver credits, and } \\
\text { health insurance }\end{array}$ & $\begin{array}{c}\text { Numerical and } \\
\text { dichotomous questions }\end{array}$ & $\begin{array}{c}\text { IV, } \\
\text { Mod/Med }\end{array}$ & $\checkmark$ & $\checkmark$ & $x$ & $x$ \\
\hline $\begin{array}{l}\text { Motivations in } \\
\text { Elder Care Scale } \\
\text { (MECS) [34] }\end{array}$ & $\begin{array}{l}\text { Two item sub-scales: } \\
\text { Extrinsic Motivations to } \\
\text { Care (EXMECS) and } \\
\text { Intrinsic Motivations to } \\
\text { Care (INMECS) }\end{array}$ & $\begin{array}{l}\text { 5-point scale ranging } \\
\text { from } 1 \text { (Strongly } \\
\text { disagree) to } 5 \text { (Strongly } \\
\text { agree). A higher score } \\
\text { indicates greater } \\
\text { motivations to provide } \\
\text { care. }\end{array}$ & $\begin{array}{c}\text { IV, DV, } \\
\text { Med, Mod }\end{array}$ & $\checkmark$ & $\boldsymbol{v}$ & $x$ & $x$ \\
\hline
\end{tabular}


Table 1. Cont.

\begin{tabular}{|c|c|c|c|c|c|c|c|}
\hline Section/Measure & $\begin{array}{l}\text { Description and } \mathrm{Max}^{*} \mathrm{~N} \\
\text { of Items }\end{array}$ & Scoring & $\begin{array}{c}\text { IV, DV, } \\
\text { Mod, Med }\end{array}$ & $\begin{array}{c}\text { CG } \\
\text { Baseline }\end{array}$ & $\begin{array}{c}\text { CG } \\
\text { Follow Up }\end{array}$ & $\begin{array}{c}\text { CR } \\
\text { BASELINE }\end{array}$ & $\begin{array}{c}\text { CR Follow } \\
\text { Up }\end{array}$ \\
\hline $\begin{array}{l}\text { Partner-Specific } \\
\text { Communal } \\
\text { Motivation Scale } \\
\text { (CMS) [35] }\end{array}$ & $\begin{array}{l}\text { Ten (10) items address } \\
\text { communal motivation to } \\
\text { care }\end{array}$ & $\begin{array}{l}\text { 9-point scale ranging } \\
\text { from } 1 \text { (Extremely } \\
\text { disagree) to } 9 \\
\text { (Extremely agree). A } \\
\text { high score reflects } \\
\text { greater CM. (items 2, } 5 \\
\text { and } 10 \text { are reverse } \\
\text { scored before } \\
\text { summing) }\end{array}$ & IV & $\checkmark$ & $\checkmark$ & $\begin{array}{c}\mathcal{V} \\
\text { adapted }\end{array}$ & $\underset{\text { adapted }}{\mathcal{V}}$ \\
\hline $\begin{array}{l}\text { Willingness to } \\
\text { Care Scale [36] }\end{array}$ & $\begin{array}{l}\text { Thirty (30) items assess } \\
\text { willingness to provide } \\
\text { emotional, instrumental, } \\
\text { and } \\
\text { nursing care related tasks }\end{array}$ & $\begin{array}{c}\text { 5-point Likert scale } \\
(1=\text { completely } \\
\text { unwilling to complete } \\
\text { the task, } 5=\text { completely } \\
\text { willing })\end{array}$ & $\begin{array}{c}\text { IV, DV, } \\
\text { Med, Mod }\end{array}$ & $\checkmark$ & $\checkmark$ & $x$ & $x$ \\
\hline $\begin{array}{l}\text { Willingness to } \\
\text { Receive Care, } \\
\text { adapted from } \\
\text { Abell [36] }\end{array}$ & $\begin{array}{c}\text { Three items assess } \\
\text { willingness to receive } \\
\text { emotional, instrumental, } \\
\text { and } \\
\text { nursing care-related tasks }\end{array}$ & $\begin{array}{c}\text { 5-point Likert scale } \\
(1=\text { completely } \\
\text { unwilling to complete } \\
\text { the task, } 5=\text { completely } \\
\text { willing })\end{array}$ & $\begin{array}{l}\text { IV, DV, } \\
\text { Med, Mod }\end{array}$ & $x$ & $x$ & $\checkmark$ & $\checkmark$ \\
\hline $\begin{array}{c}\text { The World } \\
\text { Health } \\
\text { Organisation- } \\
\text { Five Well-Being } \\
\text { Index (WHO-5) } \\
\text { [37] }\end{array}$ & $\begin{array}{c}\text { Five items assess } \\
\text { caregiver/care recipient } \\
\text { well-being }\end{array}$ & $\begin{array}{c}\text { 6-point scale ranging } \\
\text { from } 0 \text { (at no time) to } 5 \\
\text { (all of the time). High } \\
\text { scores indicate greater } \\
\text { wellbeing }\end{array}$ & DV & $\checkmark$ & $\boldsymbol{v}$ & $\checkmark$ & $\checkmark$ \\
\hline $\begin{array}{c}\text { The GAINS Scale } \\
{[38]}\end{array}$ & $\begin{array}{l}\text { Ten (10) items assess } \\
\text { perceived gains } \\
\text { associated with } \\
\text { caregiving }\end{array}$ & $\begin{array}{l}\text { Items measured on a } \\
\text { 4-point Likert scale } \\
\text { from } 0=\text { Not at all to } \\
3=\mathrm{A} \text { lot, with a } \\
\text { possible maximum } \\
\text { score of } 30 . \text { Higher } \\
\text { scores indicate greater } \\
\text { gains. }\end{array}$ & DV & $\checkmark$ & $\checkmark$ & $x$ & $x$ \\
\hline $\begin{array}{l}\text { Short Form Zarit } \\
\text { Burden Interview } \\
\text { (ZBI-12) [39] }\end{array}$ & $\begin{array}{l}\text { Twelve (12) items assess } \\
\text { caregiver burden }\end{array}$ & $\begin{array}{l}\text { 5-point Likert scale } \\
\text { ranging from } 0=\text { Never } \\
\text { to } 4=\text { Always } \\
\text { Total scores range from } \\
0 \text { to } 48 \text { with higher } \\
\text { scores }(>20) \text { indicating } \\
\text { high levels of burden }\end{array}$ & DV & $\checkmark$ & $\checkmark$ & $x$ & $x$ \\
\hline $\begin{array}{c}\text { EQ-5D-5L } \\
\text { and EQ VAS [40] }\end{array}$ & $\begin{array}{c}\text { Five dimensions of health } \\
\text { state are assessed using } 5 \\
\text { items: mobility, self-care, } \\
\text { usual activities, } \\
\text { pain/discomfort and } \\
\text { anxiety/depression. } \\
\text { The EQ VAS is a single } \\
\text { item that records the } \\
\text { patient's self-rated health } \\
\text { on a vertical "ladder" } \\
\text { visual analogue scale. }\end{array}$ & $\begin{array}{l}\text { Each dimension has } 5 \\
\text { levels: no problems, } \\
\text { slight problems, } \\
\text { moderate problems, } \\
\text { severe problems and } \\
\text { extreme problems. } \\
\text { The VAS endpoints are } \\
\text { labelled 'The best } \\
\text { imaginable health state } \\
(100)^{\prime} \text { ' and 'The worst } \\
\text { imaginable health state } \\
(0)\end{array}$ & IV, DV & $\checkmark$ & $\checkmark$ & $\checkmark$ & $\checkmark$ \\
\hline $\begin{array}{c}\text { Centre for } \\
\text { Epidemiological } \\
\text { Studies } \\
\text { Depression Scale } \\
\text { (CESD-10) } \\
{[41,42]}\end{array}$ & $\begin{array}{l}\text { This } 10 \text {-item screening } \\
\text { tool assesses depressive } \\
\text { symptoms in the past } \\
\text { week }\end{array}$ & $\begin{array}{l}\text { 4-point Likert scale } \\
\text { ranging from } 0=\text { Rarely } \\
\text { or none of the time to } \\
3=\text { All the time. } \\
\text { (items } 5 \text { and } 8 \text { are } \\
\text { reverse scored before } \\
\text { summing) }\end{array}$ & IV, DV & $\checkmark$ & $\checkmark$ & $\checkmark$ & $\checkmark$ \\
\hline
\end{tabular}


Table 1. Cont.

\begin{tabular}{|c|c|c|c|c|c|c|c|}
\hline Section/Measure & $\begin{array}{l}\text { Description and } \mathrm{Max}^{*} \mathbf{N} \\
\text { of Items }\end{array}$ & Scoring & $\begin{array}{c}\text { IV, DV, } \\
\text { Mod, Med }\end{array}$ & $\begin{array}{c}\text { CG } \\
\text { Baseline }\end{array}$ & $\begin{array}{c}\text { CG } \\
\text { Follow Up }\end{array}$ & $\begin{array}{c}\text { CR } \\
\text { BASELINE }\end{array}$ & $\begin{array}{l}\text { CR Follow } \\
\text { Up }\end{array}$ \\
\hline $\begin{array}{c}\text { Dyadic } \\
\text { Relationship } \\
\text { Scale (DRS) [43] }\end{array}$ & $\begin{array}{l}\text { The } 11 \text {-item scale for the } \\
\text { caregiver baseline and } \\
\text { follow up and the } 10 \text { item } \\
\text { scale for the care recipient } \\
\text { baseline and follow up } \\
\text { assess positive dyadic } \\
\text { interactions and negative } \\
\text { dyadic strain experienced } \\
\text { by caregivers and care } \\
\text { recipients. }\end{array}$ & $\begin{array}{c}\text { 5-point Likert scale } \\
\text { ranging from } \\
0 \text { = strongly agree to } \\
4 \text { = strongly disagree. } \\
\text { Reversed items: } \\
\text { 3,4,5,8,11. } \\
\text { Higher scores indicate } \\
\text { higher levels of strain } \\
\text { and positive } \\
\text { interaction. }\end{array}$ & DV & $\checkmark$ & $\checkmark$ & $\checkmark$ & $\checkmark$ \\
\hline $\begin{array}{l}\text { Relationship } \\
\text { satisfaction } \\
\text { (RAS) [44] }\end{array}$ & $\begin{array}{l}\text { A single item measure of } \\
\text { relationship satisfaction }\end{array}$ & $\begin{array}{l}\text { 5-point Likert scale } \\
\text { ranging from } 1=\text { not } \\
\text { satisfied to } 5=\text { very } \\
\text { satisfied }\end{array}$ & DV & $\checkmark$ & $\checkmark$ & $\checkmark$ & $\checkmark$ \\
\hline $\begin{array}{c}\text { Caregiver } \\
\text { Indirect and } \\
\text { Informal Care } \\
\text { Cost Assessment } \\
\text { Questionnaire } \\
\text { [45] }\end{array}$ & $\begin{array}{l}\text { Twelve (12) items enable } \\
\text { calculation of estimated } \\
\text { indirect (productivity) } \\
\text { and informal care costs as } \\
\text { mutually exclusive } \\
\text { subsets of total costs }\end{array}$ & & DV, IV & $\checkmark$ & $\checkmark$ & $x$ & $x$ \\
\hline
\end{tabular}

\section{Module 1}

Twenty one (21) items across three sub-scales:

ilism Scale (RFS) [46]

- Familial interconnectedness;

- Familial obligations;

- Extended family support. 5-point Likert scale, with 0 indicating 'very much in disagreement and 4 'very much in IV agreement.'

Nine items, with a single Each item assessed on a item each assessing scale from 1 to 10 illness consequences, (modified response

Brief Illness

Perception

Questionnaire

(B-IPQ) [47] meline, personal control treatment control, identity, coherence, emotional representation, and illness concern range). A summed score represents the degree to which the illness is perceived as threatening.

Five items each assess two dimensions of meaning in life:

7-point Likert scale from 1 ('absolutely untrue') to 7

Questionnaire

(1) Presence of Meaning

(MLQ) [48]

(2) Search for Meaning.

Nine items assess

Portrait Values

Questionnaire together two subscales of personal values:

(PVQ-21) [49]

Self-transcendence and Self-enhancement.

6-point Likert response

scale from "very much

like me" (1), to "not

like me at all. (6)." The subscale score is

obtained by calculating
IV

the mean of the

relevant item scores.

Perceived choice in assuming the caregiving role

Single item: Do you feel

you had a choice in

taking on this

Yes/No

IV

responsibility of caring

for your loved one?

The importance of religion

Single item: What is the importance of religion in your life? 4-point response scale

from 1 ("not important

at all") to 4 ("very important") 
Table 1. Cont.

\begin{tabular}{|c|c|c|c|c|c|c|c|}
\hline Section/Measure & $\begin{array}{l}\text { Description and Max*N } \\
\text { of Items }\end{array}$ & Scoring & $\begin{array}{l}\text { IV, DV, } \\
\text { Mod, Med }\end{array}$ & $\begin{array}{c}\text { CG } \\
\text { Baseline }\end{array}$ & $\begin{array}{c}\text { CG } \\
\text { Follow Up }\end{array}$ & $\begin{array}{c}\text { CR } \\
\text { BASELINE }\end{array}$ & $\begin{array}{c}\text { CR Follow } \\
\text { Up }\end{array}$ \\
\hline \multicolumn{8}{|c|}{ Module 2} \\
\hline $\begin{array}{l}\text { The geographies } \\
\text { of care }\end{array}$ & $\begin{array}{l}\text { Thirty (30) items for the } \\
\text { CG survey and } 8 \text { for the } \\
\text { CR identify two aspects } \\
\text { of caregiving } \\
\text { geographies; a) setting, } \\
\text { access, characteristics; } \\
\text { and b) perceived } \\
\text { geographical barriers and } \\
\text { facilitators to informal } \\
\text { care provision. }\end{array}$ & $\begin{array}{c}\text { Mixed format, Likert } \\
\text { scale responses and } \\
\text { dichotomous questions } \\
(\text { Yes/No) }\end{array}$ & IV & $\checkmark$ & $\checkmark$ & $\checkmark$ & $\checkmark$ \\
\hline $\begin{array}{l}\text { Big-Five } \\
\text { Inventory Extra } \\
\text { Short Form } \\
\text { (BFI-2-XS) [50] }\end{array}$ & $\begin{array}{l}15 \text { items measure the } \\
\text { domains of Extraversion, } \\
\text { Agreeableness, } \\
\text { Conscientiousness, } \\
\text { Neuroticism, } \\
\text { Openness to Experience }\end{array}$ & $\begin{array}{l}\begin{array}{l}5 \text {-point Likert scale } \\
\text { ranging from }\end{array} \\
1=\text { Disagree strongly } \\
\text { to } 5=\text { Agree strongly. }\end{array}$ & IV & $\checkmark$ & $\checkmark$ & $\checkmark$ & $\checkmark$ \\
\hline $\begin{array}{l}\text { The Relationship } \\
\text { Structures } \\
\text { Questionnaire of } \\
\text { the Experiences } \\
\text { in Close } \\
\text { Relationships- } \\
\text { Revised } \\
\text { (ECR-RS) [51] }\end{array}$ & $\begin{array}{l}\text { Nine items assess (1) } \\
\text { attachment-related } \\
\text { anxiety and (2) } \\
\text { attachment-related } \\
\text { avoidance }\end{array}$ & $\begin{array}{c}\text { 4-point Likert scale } \\
\text { ranging from } \\
1 \text { = Strongly Disagree } \\
\text { to } 4 \text { = Strongly } \\
\text { Agree. } \\
\text { Scores are computed } \\
\text { for each of the two } \\
\text { subscales by averaging } \\
\text { item responses. }\end{array}$ & IV & $\checkmark$ & $\checkmark$ & $\checkmark$ & $\checkmark$ \\
\hline $\begin{array}{l}\text { Toronto Empathy } \\
\text { Questionnaire } \\
\text { (TEQ) [52] }\end{array}$ & $\begin{array}{l}\text { Sixteen (16) items assess } \\
\text { empathy as a primarily } \\
\text { emotional process. }\end{array}$ & $\begin{array}{l}\text { 5-point Likert scale } \\
\text { ranging from } 0=\text { Never } \\
\text { to } 4=\text { Always. } \\
\text { Scores below } 45 \text { are } \\
\text { indicative of below } \\
\text { average empathy } \\
\text { levels. }\end{array}$ & IV & $\checkmark$ & $\checkmark$ & $\checkmark$ & $\checkmark$ \\
\hline $\begin{array}{c}\text { The Pearlin } \\
\text { Mastery Scale } \\
\text { [53] }\end{array}$ & $\begin{array}{l}\text { This seven-item scale } \\
\text { assesses the extent to } \\
\text { which an individual } \\
\text { regards their life chances } \\
\text { as being under their } \\
\text { personal control }\end{array}$ & $\begin{array}{l}\text { 4-point Likert scale } \\
\text { ranging from } \\
1=\text { Strongly Disagree } \\
\text { to } 4 \text { = Strongly Agree }\end{array}$ & IV & $\checkmark$ & $\checkmark$ & $\checkmark$ & $\checkmark$ \\
\hline \multicolumn{8}{|c|}{ Module 3} \\
\hline $\begin{array}{c}\text { Perception of } \\
\text { Collaboration } \\
\text { Questionnaire } \\
\text { (PCQ) [54] }\end{array}$ & $\begin{array}{l}\text { This nine-item scale } \\
\text { assesses three dimensions } \\
\text { of collaboration between } \\
\text { caregiver and care } \\
\text { recipient: (1) Cognitive } \\
\text { Compensation (2) } \\
\text { Interpersonal Enjoyment } \\
\text { and (3) Frequency. }\end{array}$ & $\begin{array}{l}\text { 5-point Likert scale } \\
\text { ranging from } \\
1=\text { strongly disagree to } \\
5=\text { strongly agree with } \\
\text { higher scores indicating } \\
\text { stronger agreement. } \\
\text { Items } 5 \text { and } 9 \text { are } \\
\text { reverse scored. }\end{array}$ & IV, Me & $\checkmark$ & $\checkmark$ & $\checkmark$ & $\checkmark$ \\
\hline $\begin{array}{l}\text { Dyadic Coping } \\
\text { Inventory (DCI)- } \\
\text { communication } \\
\text { subscale [55] }\end{array}$ & $\begin{array}{l}\text { The eight-item DCI } \\
\text { measures perceived } \\
\text { communication and } \\
\text { dyadic coping within a } \\
\text { close relationship when } \\
\text { one or both dyad } \\
\text { members are stressed. }\end{array}$ & $\begin{array}{l}\text { 5-point scale from } \\
1 \text { = very rarely to } \\
5 \text { = very often. } \\
\text { Subscale scores include: } \\
\text { (a) Stress } \\
\text { communicated by } \\
\text { oneself (SCO: items 1, 2, } \\
\text { 3, and 4); (b) Stress } \\
\text { communication of the } \\
\text { partner (SCP: items 5, 6, } \\
7 \text { and } 8 \text { ). }\end{array}$ & IV, Me & $\checkmark$ & $\checkmark$ & $\checkmark$ & $\checkmark$ \\
\hline
\end{tabular}


Table 1. Cont.

\begin{tabular}{|c|c|c|c|c|c|c|c|}
\hline Section/Measure & $\begin{array}{l}\text { Description and } \mathrm{Max}^{*} \mathrm{~N} \\
\text { of Items }\end{array}$ & Scoring & $\begin{array}{c}\text { IV, DV, } \\
\text { Mod, Med }\end{array}$ & $\begin{array}{c}\text { CG } \\
\text { Baseline }\end{array}$ & $\begin{array}{c}\text { CG } \\
\text { Follow Up }\end{array}$ & $\begin{array}{c}\text { CR } \\
\text { BASELINE }\end{array}$ & $\begin{array}{l}\text { CR Follow } \\
\text { Up }\end{array}$ \\
\hline $\begin{array}{l}\text { Mutuality Scale } \\
\text { (MS) [56] }\end{array}$ & $\begin{array}{l}\text { Fifteen (15) items } \\
\text { measure mutuality from } \\
\text { either the caregiver or the } \\
\text { care recipient perspective, } \\
\text { across four dimensions: } \\
\text { love and affection, shared } \\
\text { pleasurable activities, } \\
\text { shared values, and } \\
\text { reciprocity. }\end{array}$ & $\begin{array}{l}\text { 5-point Likert scale } \\
\text { ranging from } 1=\text { not at } \\
\text { all to } 4=\text { a great deal. A } \\
\text { total scale score is } \\
\text { computed by averaging } \\
\text { all item scores. }\end{array}$ & IV, Me & $\checkmark$ & $\checkmark$ & $\checkmark$ & $\checkmark$ \\
\hline $\begin{array}{l}\text { The perceived } \\
\text { partner } \\
\text { responsiveness } \\
\text { scale (PPRS) [57] }\end{array}$ & $\begin{array}{l}\text { The 12-item PPRS } \\
\text { measures the degree to } \\
\text { which people feel that } \\
\text { their significant others } \\
\text { are responsive to them. }\end{array}$ & $\begin{array}{l}\text { 9-point Likert scale } \\
\text { ranging from } 1=\text { not at } \\
\text { all true to } \\
9=\text { completely true. }\end{array}$ & IV, Me & $\checkmark$ & $\checkmark$ & $\checkmark$ & $\checkmark$ \\
\hline $\begin{array}{l}\text { Social Support } \\
\text { List (SSL) [58] }\end{array}$ & $\begin{array}{l}\text { Six items measure } \\
\text { perceived supportive } \\
\text { behaviours and seven } \\
\text { items measure perceived } \\
\text { unsupportive behaviour } \\
\text { from the caregiver and } \\
\text { the care recipient } \\
\text { perspective. }\end{array}$ & $\begin{array}{l}\text { 4-point Likert scale } \\
\text { ranging from } 1=\text { rarely } \\
\text { or never to } 4=\text { very } \\
\text { often. The scores are } \\
\text { summed into two } \\
\text { indexes, with a higher } \\
\text { score indicating a } \\
\text { higher frequency of } \\
\text { supportive and } \\
\text { unsupportive } \\
\text { behaviour. }\end{array}$ & IV, Me & $\checkmark$ & $\checkmark$ & $\checkmark$ & $\checkmark$ \\
\hline
\end{tabular}

\begin{tabular}{|c|c|c|c|c|c|c|c|}
\hline \multicolumn{8}{|c|}{ Module 4} \\
\hline $\begin{array}{l}\text { The influence of } \\
\text { informal care on } \\
\text { employment and } \\
\text { the associated } \\
\text { costs. }\end{array}$ & $\begin{array}{l}\text { Seven self-reported items } \\
\text { address the influences of } \\
\text { informal care on the } \\
\text { employment situation of } \\
\text { the caregiver, and the } \\
\text { associated costs of this. }\end{array}$ & $\begin{array}{l}\text { Categorical and } \\
\text { numerical answers }\end{array}$ & IV, DV & $\checkmark$ & $\checkmark$ & $x$ & $x$ \\
\hline $\begin{array}{l}\text { Types of home } \\
\text { care services } \\
\text { provided by paid } \\
\text { care workers }\end{array}$ & $\begin{array}{l}\text { Eighteen (18) items assess } \\
\text { which tasks and how } \\
\text { many hours of care tasks } \\
\text { (total and per type of care } \\
\text { task) are provided by } \\
\text { paid home care workers }\end{array}$ & $\begin{array}{c}\text { Categorical and } \\
\text { numerical answers. }\end{array}$ & IV, DV & $\checkmark$ & $\checkmark$ & $x$ & $x$ \\
\hline $\begin{array}{l}\text { Rationale for } \\
\text { hiring of paid } \\
\text { care workers }\end{array}$ & $\begin{array}{l}\text { Twenty two (22) items } \\
\text { assess the rationale for } \\
\text { hiring paid home care } \\
\text { workers and the decision } \\
\text { to hire or not hire } \\
\text { migrant care workers }\end{array}$ & $\begin{array}{c}\text { Categorical answers, } \\
\text { with each item treated } \\
\text { separately }\end{array}$ & DV & $\checkmark$ & $\checkmark$ & $\checkmark$ & $\checkmark$ \\
\hline $\begin{array}{l}\text { Out-of-pocket } \\
\text { expenses } \\
\text { incurred by } \\
\text { caregivers as a } \\
\text { result of their } \\
\text { caregiving role. }\end{array}$ & $\begin{array}{l}\text { Twenty five (25) items } \\
\text { measure out-of-pocket } \\
\text { costs for caregivers (both } \\
\text { in terms of overall total } \\
\text { and per type of cost). } \\
\text { These costs include } \\
\text { expenditure on, e.g., } \\
\text { caregiving support } \\
\text { services, medical care, } \\
\text { food, travel, home care } \\
\text { services, aids, appliances } \\
\text { and home modifications } \\
\text { due to care recipient's } \\
\text { condition. }\end{array}$ & $\begin{array}{l}\text { Categorical and } \\
\text { numerical answers. }\end{array}$ & IV, DV & $\checkmark$ & $\checkmark$ & $x$ & $x$ \\
\hline
\end{tabular}


Table 1. Cont.

\begin{tabular}{|c|c|c|c|c|c|c|c|}
\hline Section/Measure & $\begin{array}{l}\text { Description and } \operatorname{Max}^{*} \mathbf{N} \\
\text { of Items }\end{array}$ & Scoring & $\begin{array}{c}\text { IV, DV, } \\
\text { Mod, Med }\end{array}$ & $\begin{array}{c}\mathrm{CG} \\
\text { Baseline }\end{array}$ & $\begin{array}{c}\text { CG } \\
\text { Follow Up }\end{array}$ & $\begin{array}{c}\text { CR } \\
\text { BASELINE }\end{array}$ & $\begin{array}{c}\text { CR Follow } \\
\text { Up }\end{array}$ \\
\hline $\begin{array}{l}\text { Care benefits } \\
\text { received by the } \\
\text { care recipient }\end{array}$ & $\begin{array}{l}\text { Four self-reported items } \\
\text { measure care benefits } \\
\text { received (both in terms of } \\
\text { overall total and per type } \\
\text { of care benefit), e.g., tax } \\
\text { benefits, cash benefits, } \\
\text { care vouchers. }\end{array}$ & $\begin{array}{c}\text { Categorical and } \\
\text { numerical answers. }\end{array}$ & IV, DV & $x$ & $x$ & $\checkmark$ & $\checkmark$ \\
\hline $\begin{array}{l}\text { Self-reported } \\
\text { questionnaire on } \\
\text { the use of, and } \\
\text { the out-of-pocket } \\
\text { expenses for, care } \\
\text { services, as well } \\
\text { as assistive } \\
\text { devices and aids } \\
\text { used by the care } \\
\text { recipient. }\end{array}$ & $\begin{array}{l}\text { Twenty two (22) items } \\
\text { detail which types of } \\
\text { support services the care } \\
\text { recipient receives in } \\
\text { relation to their care and } \\
\text { the out of pocket } \\
\text { expenses spent in relation } \\
\text { to their care. }\end{array}$ & $\begin{array}{c}\text { Categorical and } \\
\text { numerical answers }\end{array}$ & IV, DV & $x$ & $x$ & $\checkmark$ & $\checkmark$ \\
\hline
\end{tabular}

Legend: $\mathrm{CG}=$ caregiver; $\mathrm{CR}=$ care recipient; $\mathrm{IV}=$ independent variable; $\mathrm{DV}=$ dependent variable; $\mathrm{Mo}=$ moderator $\mathrm{Me}=$ mediator .

Measures that are not validated and available in the required language will be translated into the appropriate languages using accredited translators who will be native speakers of the target languages and fluent in English. Translations will be checked for compatibility with the original version in a process of back translation, also performed by persons who will be native in the foreign language and fluent in the English language, to ensure that none of the original meaning is lost. For each language, a potential research consultant/reviewer will be identified to ensure that any discrepancies between the forward and back translations can be resolved appropriately by discussion with the translators. All translations will be coordinated by one project partner to ensure consistency. Piloting in each country shall enable identification of any semantic inconsistencies.

\section{Analysis Plan}

Five separate studies are addressed, and each will use selected variables from the Core survey, plus module-specific items. For each study, baseline data will first be analysed for the full sample, with a smaller sample entering longitudinal analysis dependent on completing both timepoints of the survey. The analysis plan will evolve depending on our a priori set of research questions, as well as the sample characteristics and final sample size.

Missing data handling. Prior to analysis the data set will be cleansed and checked for missing data. Missing data are a critical challenge when dealing with online surveys thus the ENTWINE iCohort survey will employ multiple checkpoints to address this. In the cases where missing data exist, a descriptive analysis of the missing data, as well as a number of hypothesis tests, will be carried out to determine the mechanism of missingness. These tests include Little's test and Fairclough's logistic regression method $[59,60]$. If data are missing completely at random (MCAR), missing values will be subjected to list-wise deletion and complete case analysis will be performed. If data is missing at random (MAR), we will adopt a hot deck imputation for variables with a negligible proportion of missing values (i.e., less than 5\%) and multiple imputations by chained equations (MICE) for variables with a larger proportion of missing values [61-64].

For all analyses, assumptions regarding multicollinearity, singularity, normality, linearity, and homoscedasticity will be tested.

\subsection{Modules 1-2-3 Plan Analyses}

Descriptive analyses will be performed to establish frequencies, means and standard deviations across key categorical and continuous variables of interest. Bivariate and multivariate analyses, including analysis of variance, $t$-tests and correlations will examine 
the relationship between caregiver and care recipient characteristics (e.g., age, gender, ethnicity, illness type, type of caregiving relationship, etc.), willingness and motivations to provide care, and key caregiver and care recipient outcomes (e.g., caregiver burden, depression, relationship satisfaction etc.).

Relevant, theoretically informed tests of mediation and moderation will be applied prior to conducting hierarchical multivariate regression analyses to assess the extent of outcome variance explained by the IV set, for example moderating or mediating the effects of: (1) relationship quality, gender, or perceived choice in assuming the caregiver role; (2) willingness to care and relationship quality of the caregiving dyad; (3) interpersonal processes and type of relationship between caregiver and care recipient.

Changes over time in IVs or caregiving outcomes will be analysed in the longitudinal data set using repeated measures tests ( $t$-tests, ANOVA, MANOVA).

\subsection{Module 4 Plan Analysis}

Descriptive analyses will be conducted with sociodemographic and caregiving situation data to summarise sample characteristics, informal care costs and the use of paid home care services.

Informal care costs including employment-related costs, caregiver-time costs, and outof-pocket expenses will be estimated. Unlike out-of-pocket costs, time- and employmentrelated costs are not measured in monetary terms and, therefore, should be valued. Time costs will be valued by two methods: the opportunity costs and the proxy good (following the recommendation in van den Berg et al. [65]). Additionally, employment-related costs will be valued using the human capital approach. Additional informal caregivers, the underlying level of dependency (i.e., need) of, and distance to, the care recipient may mediate the time and burden perceived. During analyses these interactions will be explored. Furthermore, multivariate regression analysis will be performed to find the determinants of informal care costs, e.g., those mentioned above, the demographic and socioeconomic characteristics of both the caregiver and care recipient, as well as the use of national or local formal care and support services.

The impact of the hiring of paid care workers (alongside other factors) on the amount of informal care will be examined using bivariate and linear regression. Furthermore, binary logistic regression will be used to calculate the predictors (including the hiring of paid care workers) of not having a low level of subjective wellbeing (score of 50 or over on the WHO-5 index). Finally, hours of personal care, household activities, and practical support by informal caregivers and paid care workers will be cross tabulated with each other to determine if care tasks are provided by both informal caregivers and paid care workers, only provided by paid care workers or informal caregivers, or provided by neither.

\section{Weekly Assessment Component}

In the weekly assessment component, multilevel modelling will be used to account for the nested data structure (i.e., weekly diary assessments nested within caregivers [66]) in order to assess within-person main, interaction and moderation effects. Intensive longitudinal designs (e.g., weekly assessments) can better provide critical information on fluctuations in willingness to care, and identify how they are associated with changes in care tasks, caregiver burden or mood and in interpersonal processes between caregivers and care recipients (e.g., dyadic coping behaviours). These data will examine time-lagged associations between key factors and caregiver outcomes in order to gain better insight into the sequence of events and address the predictive utility of our measures in relation to key outcomes. Our analyses will tease apart the specific challenges faced by informal caregivers at different stages of the disease of the care recipient, i.e., during different periods in their caregiving role, and thus help to target support (e.g., eHealth) at the appropriate time. 


\section{Dissemination}

A dissemination strategy has been prepared by the ENTWINE WorkPackage group and our Dissemination and Website Taskforce. This will include dissemination to both academic and non-academic stakeholders in health, public health and social care sectors by means of conference presentations, blogs and multi-media news items on our study website, and preparation of a newsletter providing an executive summary of study findings and their implications.

Study status-Baseline recruitment to 31 August 2021; recruitment figures 2736 recruited, 1834 completers (1120 full completion) with follow-up ongoing.

Ireland $14 / 08 / 2020-31 / 05 / 2021$

UK 14/08/2020-31/05/2021

Poland $14 / 08 / 2020-31 / 05 / 2021$

Italy $25 / 08 / 2020-31 / 05 / 2021$

Netherlands 16/10/2020-31/05/2021

Sweden 23/10/2020-31/05/2021

Greece 31/10/2020-31/05/2021

Israel 16/02/2021-31/08/2021

Germany 17/02/2021-31/08/2021

Participants were invited to take part in the follow up assessment 181 days after the initial baseline invitation. Data collection completed for all countries on 15 December 21 6 month follow-ups were not offered to participants who completed baseline data after 31 May 2021 due to project funding deadline restrictions.

Supplementary Materials: The following are available online at https:/ /www.mdpi.com/article/10 .3390/ijerph19020821/s1, Supplementary File S1: ENTWINE-iCohort exemplary fliers (1-3); Supplementary File S2: Participant Information Sheet for ENTWINE-iCohort Prospective Study in Informal Caregiving; Supplementary File S3: Participant Consent Forms to participate in the survey; Supplementary File S4: ENTWINE-iCOHORT Caregiver Baseline Survey; Supplementary File S5: ENTWINE-iCOHORT Care Recipient Baseline Survey; Supplementary File S6: ENTWINE-iCOHORT Caregiver Follow up Survey; Supplementary File S7: ENTWINE-iCOHORT Care Recipient Follow up Survey; Supplementary File S8: ENTWINE-iCOHORT Diary Study Weekly Measures-Caregiver version; Supplementary File S9: ENTWINE-iCOHORT Diary Study Weekly Measures-Care Recipient; Supplementary File S10: Consent to be contacted for future studies; Supplementary File S11: Participant Consent Form to be contacted for future studies; Eligibility Survey.

Author Contributions: Conceptualization, V.M., N.V., R.S., M.H., developed the ENTWINE funding proposal to MSC ITN of which the iCohort survey design and objectives comprises WorkPackage 1; Protocol Methodology, V.M., N.V., M.H., R.S., G.L., E.B. (Erik Buskens); E.B. (Eva Bei), M.Z., S.E., O.F., G.F.; software, SE.; Investigation, V.M., N.V., M.H., G.L., E.B. (Erik Buskens); E.B. (Eva Bei), M.Z., S.E., O.F., G.F.; Resources, V.M., N.V., M.H., G.L., E.B. (Erik Buskens); A.L.; Data curation, M.H., V.M., N.V., G.L., E.B. (Erik Buskens), Writing—original draft preparation, V.M.; Writing-review and editing, M.Z., N.V., O.F., G.F., E.B. (Eva Bei), V.A., S.E., E.B. (Erik Buskens), G.L., and M.H., supervision, V.M., M.H., N.V., E.B. (Erik Buskens), G.L., project administration, M.H., V.M., N.V., G.L., E.B. (Erik Buskens); A.L.; funding acquisition, M.H., V.M., N.V., E.B. (Erik Buskens), G.L. All authors have read and agreed to the published version of the manuscript.

Funding: This research project is funded by the European Union's Horizon 2020 research and innovation programme under the Marie Skłodowska-Curie Innovative Training Network (H2020MSCA-ITN-2018), grant agreement No. 814072. In addition, one ESR has received partial support through Ricerca Corrente funding from the Italian Ministry of Health to IRCCS INRCA.

Institutional Review Board Statement: The study was conducted according to the guidelines of the Declaration of Helsinki $(1975,2013)$, and primary full approval obtained from the Institutional Review Board (or Ethics Committee) of Bangor University for non-clinical recruitment and NHS Research Ethics and Governance Committee for clinical site recruitment (protocol code 20/WA/0006, January and June 2020, respectively). Subsequent to this, the English language documents were translated and submitted to the other participating countries as required by national legislation, between January and 
October 2020 with several COVID-related delays. Central Ethics Review Board non-WMO studies, University Medical Centre Groningen, The Netherlands, Ref No. 201900810. Bar-Ilan University, Faculty of Social Sciences, Department of Psychology, Ethics Committee, Ramat Gan, Israel Ref No: 36-20. Commissione Etica per la Ricerca in Psicologia (CERPS), Università Cattolica del Sacro Cuore di Milano, Italy, Ref No. 31-20. Swedish Ethical Review Authority, Uppsala University, Sweden, Ref No.: 2020-04569. Medical Ethics Committee, Department of Health Services Research, University of Oldenburg, Oldenburg, Germany. Ref.No. 2020-155. Ethical Approval Confirmation conferred via the Institute of Psychology at the University of Wroclaw in Poland on basis of the UK NHS Research Ethics Approval. Approval in Greece conferred via the University Medical Centre Groningen in The Netherlands on basis of approvals from other European nations. In Ireland organizations that assisted in disseminating the study acknowledged ethics approval from other countries, for example UK NHS Research Ethics Committee approval. i.e., nation-specific ethical review and approval were waived for this study.

Informed Consent Statement: Informed consent was obtained from all study participants. All participants provided informed electronic consent and could not proceed in the online survey system without having indicated this.

Data Availability Statement: This is a protocol paper however all anonymized data will be openly available upon request after September 2023, or on completion of ENTWINE teams key outputs if this is earlier. OpenAIRE and Zenodo will be used as data repositories.

Acknowledgments: Thanks to UMCG administrative and technical support teams for training and support in the use of QUESTBACK.

Conflicts of Interest: The authors declare no conflict of interest. The funders had no role in the design of the protocol for this study; in the writing of the manuscript, or in the decision to publish the results.

\section{References}

1. The Health Foundation. Health and Social Care Funding Explained; The Health Foundation: London, UK, 2017.

2. Arcà, E.; Principe, F.; Van Doorslaer, E. Death by austerity? The impact of cost containment on avoidable mortality in Italy. Health Econ. 2020, 29, 1500-1516. [PubMed]

3. Carers UK. State of Caring. A Snapshot of Unpaid Care in the UK; Carers UK: London, UK, 2019.

4. Tur-Sinai, A.; Teti, A.; Rommel, A.; Hlebec, V.; Lamura, G. How many older informal caregivers are there in Europe? Comparison of estimates of their prevalence from three european surveys. Int. J. Environ. Res. Public Health 2020, $17,9531$.

5. Rodrigues, R.; Huber, M.; Lamura, G. Facts and Figures on Healthy Ageing and Long-Term Care; Citeseer: Vienna, Austria, 2012.

6. Ortiz-Ospina, E.; Tzvetkova, S.; Roser, M. Women's Employment. Available online: https://ourworldindata.org/female-laborsupply (accessed on 15 October 2021).

7. Greenwood, N.; Smith, R. Motivations for being informal carers of people living with dementia: A systematic review of qualitative literature. BMC Geriatr. 2019, 19, 1-18. [CrossRef]

8. Morrison, V.; Williams, K. Gaining Longitudinal Accounts of Carers' Experiences Using IPA and Photograph Elicitation. Front. Psychol. 2020, 11, 2429.

9. Zarzycki, M.; Morrison, V. Getting back or giving back: Understanding caregiver motivations and willingness to provide informal care. Health Psychol. Behav. Med. 2021, 9, 636-661. [PubMed]

10. Berg, C.A.; Upchurch, R. A developmental-contextual model of couples coping with chronic illness across the adult life span. Psychol. Bull. 2007, 133, 920.

11. Dagan, M.; Sanderman, R.; Schokker, M.C.; Wiggers, T.; Baas, P.C.; Van Haastert, M.; Hagedoorn, M. Spousal support and changes in distress over time in couples coping with cancer: The role of personal control. J. Fam. Psychol. 2011, 25, 310.

12. Falconier, M.K.; Kuhn, R. Dyadic coping in couples: A conceptual integration and a review of the empirical literature. Front. Psychol. 2019, 10, 571. [PubMed]

13. Hagedoorn, M.; Puterman, E.; Sanderman, R.; Wiggers, T.; Baas, P.C.; van Haastert, M.; DeLongis, A. Is self-disclosure in couples coping with cancer associated with improvement in depressive symptoms? Health Psychol. 2011, 30, 753.

14. Vilchinsky, N.; Dekel, R.; Revenson, T.A.; Liberman, G.; Mosseri, M. Caregivers' burden and depressive symptoms: The moderational role of attachment orientations. Health Psychol. 2015, 34, 262.

15. Parveen, S.; Morrison, V.; Robinson, C.A. Ethnic variations in the caregiver role: A qualitative study. J. Health Psychol. 2011, 16, 862-872. [CrossRef]

16. Zarzycki, M.; Morrison, V.; Bei, E.; Seddon, D. Cultural and societal motivations for being informal caregivers: A qualitative systematic review and meta-synthesis. Health Psychol. Rev. 2021, in press. 
17. Casanova, G.; Di Rosa, M.; Fisher, O.; Lamura, G. Between migrant care work and new occupational welfare tools: Changing home care arrangements in Italy. Int. J. Environ. Res. Public Health 2020, 17, 5511.

18. Baldassar, L.; Baldock, C.V.; Wilding, R. Families Caring across Borders: Migration, Ageing and Transnational Caregiving; Springer: Berlin/Heidelberg, Germany, 2006; ISBN 0230626262.

19. Bei, E.; Zarzycki, M.; Morrison, V.; Vilchinsky, N. Motivations and willingness to provide care from a geographical distance, and the impact of distance care on caregivers' mental and physical health: A mixed-method systematic review protocol. BMJ Open 2021, 11, 1-7. [CrossRef]

20. Bei, E.; Rotem-Mindali, O.; Vilchinsky, N. Providing Care from Afar: A Growing Yet Understudied Phenomenon in the Caregiving Field. Front. Psychol. 2020, 11, 10-12. [CrossRef]

21. Douglas, S.L.; Mazanec, P.; Lipson, A.; Leuchtag, M. Distance caregiving a family member with cancer: A review of the literature on distance caregiving and recommendations for future research. World J. Clin. Oncol. 2016, 7, 214.

22. Li, M.; Mao, W.; Chi, I.; Lou, V.W.Q. Geographical proximity and depressive symptoms among adult child caregivers: Social support as a moderator. Aging Ment. Health 2019, 23, 205-213.

23. Krol, M.; Papenburg, J.; van Exel, J. Does including informal care in economic evaluations matter? A systematic review of inclusion and impact of informal care in cost-effectiveness studies. Pharmacoeconomics 2015, 33, 123-135.

24. Anderson, A. Europe's care regimes and the role of migrant care workers within them. J. Popul. Ageing 2012, 5, 135-146. [PubMed]

25. Pinquart, M.; Sorensen, S. Ethnic differences in stressors, resources, and psychological outcomes of family caregiving: A meta-analysis. Gerontologist 2005, 45, 90-106. [CrossRef] [PubMed]

26. Verbakel, E. How to understand informal caregiving patterns in Europe? The role of formal long-term care provisions and family care norms. Scand. J. Public Health 2018, 46, 436-447. [PubMed]

27. Bom, J.; Bakx, P.; Schut, F.; Van Doorslaer, E. Health effects of caring for and about parents and spouses. J. Econ. Ageing 2019, 14, 100196.

28. Carmichael, F.; Charles, S.; Hulme, C. Who will care? Employment participation and willingness to supply informal care. J. Health Econ. 2010, 29, 182-190. [CrossRef] [PubMed]

29. Pickard, L. A growing care gap? The supply of unpaid care for older people by their adult children in England to 2032. Ageing Soc. 2015, 35, 96-123.

30. Kooiker, S.; de Jong, A.; Verbeek-Oudijk, D.; de Boer, A. Toekomstverkenning Mantelzorg Aan Ouderen in 2040; SCP: London, UK, 2019.

31. Faul, F.; Erdfelder, E.; Buchner, A.; Lang, A.-G. Statistical power analyses using G* Power 3.1: Tests for correlation and regression analyses. Behav. Res. Methods 2009, 41, 1149-1160.

32. Aron, A.; Aron, E.N.; Smollan, D. Inclusion of other in the self scale and the structure of interpersonal closeness. J. Pers. Soc. Psychol. 1992, 63, 596.

33. Katz, S. Assessing Self-Maintenance: Activities of Daily Living, Mobility, and Instrumental Activities of Daily Living. J. Am. Geriatr. Soc. 1983, 31, 721-727.

34. Lyonette, C.; Yardley, L. The influence on carer wellbeing of motivations to care for older people and the relationship with the care recipient. Ageing Soc. 2003, 23, 487-506. [CrossRef]

35. Lemay, E.P., Jr.; Neal, A.M. The wishful memory of interpersonal responsiveness. J. Pers. Soc. Psychol. 2013, 104, 653.

36. Abell, N. Assessing Willingness to Care for Persons with AIDS: Validation of a New Measure. Res. Soc. Work Pract. 2001, 11, 118-130. [CrossRef]

37. Hermanns, N. WHO-5-Well-Being-Index. Der Diabetol. 2007, 3, 464-465.

38. Pearlin, L.I. Caregiver's Stress and Coping Study; University of California: San Francisco, CA, USA, 1988.

39. Bédard, M.; Molloy, D.W.; Squire, L.; Dubois, S.; Lever, J.A.; O’Donnell, M. The Zarit Burden Interview: A new short version and screening version. Gerontologist 2001, 41, 652-657. [PubMed]

40. Herdman, M.; Gudex, C.; Lloyd, A.; Janssen, M.F.; Kind, P.; Parkin, D.; Bonsel, G.; Badia, X. Development and preliminary testing of the new five-level version of EQ-5D (EQ-5D-5L). Qual. life Res. 2011, 20, 1727-1736. [PubMed]

41. Radloff, L.S. The CES-D scale: A self-report depression scale for research in the general population. Appl. Psychol. Meas. 1977, 1, 385-401.

42. Miller, W.C.; Anton, H.A.; Townson, A.F. Measurement properties of the CESD scale among individuals with spinal cord injury. Spinal Cord 2008, 46, 287-292. [PubMed]

43. Sebern, M.D.; Whitlatch, C.J. Dyadic relationship scale: A measure of the impact of the provision and receipt of family care. Gerontologist 2007, 47, 741-751. [CrossRef] [PubMed]

44. Hendrick, S.S.; Dicke, A.; Hendrick, C. The relationship assessment scale. J. Soc. Pers. Relat. 1998, 15, 137-142.

45. Landfeldt, E.; Zethraeus, N.; Lindgren, P. Standardized questionnaire for the measurement, valuation, and estimation of costs of informal care based on the opportunity cost and proxy good method. Appl. Health Econ. Health Policy 2019, 17, 15-24.

46. Losada, A.; Márquez-González, M.; Vara-García, C.; Barrera-Caballero, S.; Cabrera, I.; Gallego-Alberto, L.; Olmos, R.; RomeroMoreno, R. Measuring familism in dementia family caregivers: The revised familism scale. Aging Ment. Health 2019, $24,784-788$. [CrossRef]

47. Broadbent, E.; Petrie, K.J.; Main, J.; Weinman, J. The brief illness perception questionnaire. J. Psychosom. Res. 2006, 60, 631-637. 
48. Steger, M.F.; Frazier, P.; Kaler, M.; Oishi, S. The meaning in life questionnaire: Assessing the presence of and search for meaning in life. J. Couns. Psychol. 2006, 53, 80-93. [CrossRef]

49. Schwartz, S.H. A proposal for measuring value orientations across nations. Quest. Packag. Eur. Soc. Surv. 2003, $259,261$.

50. Soto, C.J.; John, O.P. Short and extra-short forms of the Big Five Inventory-2: The BFI-2-S and BFI-2-XS. J. Res. Pers. 2017, 68, 69-81.

51. Fraley, R.C.; Heffernan, M.E.; Vicary, A.M.; Brumbaugh, C.C. The experiences in close relationships-Relationship Structures Questionnaire: A method for assessing attachment orientations across relationships. Psychol. Assess. 2011, 23, 615. [PubMed]

52. Spreng, R.N.; McKinnon, M.C.; Mar, R.A.; Levine, B. The Toronto Empathy Questionnaire: Scale Development and Initial Validation of a Factor-Analytic Solution to Multiple Empathy Measures. J. Pers. Assess. 2009, 91, 62-71. [PubMed]

53. Pearlin, L.I.; Schooler, C. The structure of coping. J. Health Soc. Behav. 1978, 19, 2-21. [PubMed]

54. Berg, C.A.; Schindler, I.; Maharajh, S. Adolescents' and mothers' perceptions of the cognitive and relational functions of collaboration and adjustment in dealing with type 1 diabetes. J. Fam. Psychol. 2008, 22, 865. [PubMed]

55. Bodenmann, G. Stress und Coping Bei Paaren [Stress and Coping in Couples]; Hogrefe: Göttingen, Germany, $2000 ;$ ISBN 3801713520.

56. Archbold, P.G.; Stewart, B.J.; Greenlick, M.R.; Harvath, T. Mutuality and preparedness as predictors of caregiver role strain. Res. Nurs. Health 1990, 13, 375-384. [PubMed]

57. Perceived Partner Responsiveness Scale (PPRS) (Reis \& Carmichael, 2006). Available online: https://onlinelibrary.wiley.com/ doi/pdf/10.1002/9781119102991.ch57 (accessed on 15 August 2021).

58. Kempen, G.; Van Eijk, L.M. The psychometric properties of the SSL12-I, a short scale for measuring social support in the elderly. Soc. Indic. Res. 1995, 35, 303-312.

59. Fairclough, D.L. Design and Analysis of Quality of Life Studies in Clinical Trials; CRC Press: Boca Raton, FL, USA, 2010. ISBN 1420061186.

60. Little, R.J.A. A test of missing completely at random for multivariate data with missing values. J. Am. Stat. Assoc. 1988, 83, 1198-1202.

61. Van Buuren, S. Flexible Imputation of Missing Data; CRC Press: Boca Raton, FL, USA, 2018. ISBN 0429960352.

62. Myers, T.A. Goodbye, listwise deletion: Presenting hot deck imputation as an easy and effective tool for handling missing data. Commun. Methods Meas. 2011, 5, 297-310.

63. Rubin, D.B. Multiple Imputation for Non Response in Surveys; Wiley: New York, NY, USA, 1987.

64. White, I.R.; Royston, P.; Wood, A.M. Multiple imputation using chained equations: Issues and guidance for practice. Stat. Med. 2011, 30, 377-399. [PubMed]

65. Van den Berg, B.; Brouwer, W.B.F.; Koopmanschap, M.A. Economic valuation of informal care. Eur. J. Health Econ. Former. HEPAC 2004, 5, 36-45.

66. Bolger, N.; Laurenceau, J.-P. Intensive Longitudinal Methods: An Introduction to Diary and Experience Sampling Research; Guilford Press: New York, NY, USA, 2013. ISBN 146250678X. 